\title{
Optimal Search Strategy for Finding Transients in Large-sky Error Regions under Realistic Constraints
}

\author{
Javed Rana ${ }^{1}$ (1), Shreya Anand ${ }^{2}$, and Sukanta Bose ${ }^{1,3}$ \\ ${ }^{1}$ Inter-University Centre for Astronomy and Astrophysics, Post Bag 4, Ganeshkhind, Pune 411 007, India; javed@iucaa.in \\ ${ }^{2}$ Division of Physics, Math, and Astronomy, California Institute of Technology, Pasadena, CA 91125, USA \\ ${ }^{3}$ Department of Physics and Astronomy, Washington State University, 1245 Webster, Pullman, WA 99164-2814, USA \\ Received 2019 February 19; revised 2019 April 2; accepted 2019 April 3; published 2019 May 8
}

\begin{abstract}
In order to identify the rapidly fading, optical transient counterparts of gravitational-wave (GW) sources, an efficient follow-up strategy is required. Since most ground-based optical telescopes aimed at following up GW sources have a small field of view (FOV) as compared to the GW sky error region, we focus on a search strategy that involves dividing the GW patch into tiles of the same area as the telescope FOV to strategically image the entire patch. We present an improvement over the optimal algorithm outlined in Rana et al. by factoring in the effects of air mass, telescope slew, and setting constraints into the scheduling algorithm in order to increase the chances of identifying the GW counterpart. We propose two separate algorithms: the air-mass-weighted algorithm, a solution to the Hungarian algorithm that maximizes probability acquired while minimizing the image air mass, and the slew-optimization algorithm that minimizes the overall slew angle within the observation schedule using the traveling salesman algorithm. We simulate hundreds of telescope-patch configurations to test the performance of our algorithms. Our results indicate that slew optimization can reduce the cumulative slew angle by hundreds of degrees, saving minutes of observation time without any loss of probability. Further, we demonstrate that, as compared to the greedy algorithm, the air-mass-weighted algorithm can acquire up to $20 \%$ more probability and $30 \mathrm{deg}^{2}$ more in areal coverage for skymaps of all sizes and configurations. Our analysis can be straightforwardly extended to optical counterparts of gamma-ray bursts, as well as to other telescopes or sites.
\end{abstract}

Key words: gravitational waves - methods: observational - telescopes

\section{Introduction}

Within the next few years of gravitational-wave (GW) astrophysics, current estimates predict the identification of tens of compact binary coalescence (CBC) sources with electromagnetic (EM) counterparts detectable by ground-based optical telescopes (Abbott et al. 2018). Specifically, among CBC sources, the most promising candidates to contain a visible electromagnetic (EM) counterpart are binary neutron star (BNS) and neutron star-black hole (NSBH) systems. The recent detection of the BNS system GW170817 using the Hanford-Livingston-Virgo detector network (Abbott et al. 2017a) demonstrated that a wealth of information can be gained from combining the electromagnetic and $\mathrm{GW}$ emission from the same source. For the 2017 detection, GW-EM information was used to identify the source host galaxy (Abbott et al. 2017a), probe properties of the progenitor and remnant (Abbott et al. 2017d, 2017e), estimate the Hubble constant (Abbott et al. 2017b), constrain possible models for the merger and emission (Abbott et al. 2017c), and study the $r$-process nucleosynthesis processes resulting from the collision of the two neutron stars (Kasliwal et al. 2017; Smartt et al. 2017; Coughlin et al. 2018). Optical and infrared observation of GW170817 also marked the first confirmed detection of a kilonova, the bright, infrared or UV emission hypothesized to result from the $r$-process nucleosynthesis occurring during a BNS or NSBH merger (Andreoni et al. 2017; Arcavi et al. 2017; Chornock et al. 2017; Coulter et al. 2017; Cowperthwaite et al. 2017; Drout et al. 2017; Evans et al. 2017; Hu et al. 2017; Kasliwal et al. 2017; Lipunov et al. 2017; McCully et al. 2017; Pian et al. 2017; Smartt et al. 2017; Tanvir et al. 2017; Shappee et al. 2017). This study focuses on the problem of identifying optical counterparts to GW or gamma-ray bursts (GRBs) localized to large sky error regions.

Due to the proximity of the BNS system to Earth, the GW source was localized to a $90 \%$ sky area and volume of $28 \mathrm{deg}^{2}$ and $380 \mathrm{Mpc}^{3}$, respectively, making it the most well localized among all past GW detections (Abbott et al. 2017a). The fact that the source was localized to the nulls of Virgo's antenna pattern significantly reduced the localization area from an ordinary two-detector localization (Abbott et al. 2017a). IM2H, the observing team that first detected the optical counterpart to GW170817 about $10 \mathrm{hr}$ after the GW detection, employed a strategy of targeting known galaxies within the source's localization volume (Kilpatrick et al. 2017). Though we anticipate that some future BNS detections will be as nearby and well localized as GW170817, not all the GW detections of BNS or NSBH systems will have a detectable bright optical counterpart. In fact, only a small fraction of sources will be optimally oriented, maximizing the GW amplitude and favoring the detection of a coincident on-axis afterglow (Metzger \& Berger 2012; Barnes \& Kasen 2013; Petrillo et al. 2013; Kasen et al. 2015; Metzger 2017). Thus, we do not anticipate the search for optical counterparts to BNS sources to be as straightforward as it was during the GW170817 detection. However, the kilonova emission that is expected to accompany almost all BNS and NSBH mergers (with the black hole mass within $\sim 10 M_{\odot}$ ) is expected to be more isotropic than a GRB afterglow, especially in the case of an NSBH merger (Metzger 2017). Even in cases where no coincident GRB is visible owing to the viewing angle, it may be possible to extensively search the GW localization and identify the kilonova counterpart. 
The approach for detecting counterparts to GW events differs based on the wavelength of the emission, due to the fact that each type of emission is observable for a different duration of time. Optical counterparts to BNS and NSBH sources, which could either be kilonovae (Metzger 2017) or optical afterglows (Ghosh \& Bose 2013; Ascenzi et al. 2019), fade rapidly and could last anywhere from hours to days after the GW source is detected (Metzger \& Berger 2012). Using optical telescopes to observe the source as early as possible after the GW detection will maximize the information gained from the source spectrum. During the current third GW observing run, the two advanced LIGO (Aasi et al. 2015) interferometers and the advanced Virgo (Acernese et al. 2014) interferometer will be "online" and taking data for $1 \mathrm{yr}$. Based on the expected number and sensitivity of GW detectors detecting BNS and NSBH sources in this Advanced Detector Era (ADE), GW localization sky error regions are expected to span a few tens to a few hundreds of square degrees (Nissanke et al. 2013; Singer et al. 2014), while most wide-field optical observatories following up $\mathrm{GW}$ sources tend to have fields of view (FOVs) of less than a degree to a few square degrees, requiring an extensive search within the sky localization region in order to locate the optical transient (Singer et al. 2014; Cornish \& Littenberg 2015; Essick et al. 2015; Klimenko et al. 2016; Rana et al. 2017). Modern optical surveys with large FOV telescopes such as ZTF (Bellm 2014), ATLAS (Shanks et al. 2015), PanSTARRS (Chambers et al. 2016), and LSST (Ivezić et al. 2019) are considered ideal in this scenario; however, implementing optimized search strategies for smaller-FOV optical telescopes will increase the odds of identifying a coincident optical counterpart to a given BNS/NSBH trigger by strengthening the overall telescope follow-up network. In the remainder of the paper, our discussion of "optical telescopes" will primarily concern telescopes for which a scheduling strategy is most relevant (i.e., FOV $<10 \mathrm{deg}^{2}$ ), though the same methods could be applied to optimize the scheduling of larger-FOV telescopes.

Optical telescopes usually employ either the galaxy-targeted or the tiling strategies to search for GW counterparts. The former strategy requires identification of all of the galaxies within the sky error region (and therefore a near-complete galaxy catalog in the vicinity of the source) and determines the probability of the galaxy containing the GW trigger, based on its mass or luminosity. Then, ranking the galaxies in order of their properties, the algorithm will provide the telescope with the coordinates of each of the galaxies within the region and the order in which the telescope should point at them (Gehrels et al. 2016).

The tiling strategy has four main steps: placing tiles, allocating time, scheduling, and evaluating efficiency (Coughlin et al. 2018). Among different tiling methods described in Coughlin et al. (2018), the ranked tiling method (Ghosh et al. 2016) that we employ in this paper involves dividing the GW sky error region into "tiles" that are the size and shape of the FOV of the observing telescope. After the placement of tiles, the next step is to perform time allocation. Salafia et al. (2017) and Chan et al. (2017) propose time allocation based on counterpart light-curve models, while Coughlin \& Stubbs (2016) derive scaling relations for time allocation based on GW likelihood and galactic extinction. The two algorithms we present in this work use two different methods of allocating exposure time to tiles. Once exposure time has been allocated to each tile, one can schedule the tiles for observation. Scheduling involves running an optimization algorithm on the tiles to maximize probability and patch area coverage and generating a list of the coordinates of the observable tiles and the order in which the telescope should observe them (Rana et al. 2017). Finally, one can evaluate the process by running simulations and determining the amount of GW probability acquired by tiling the patch. Our optimization algorithms combine the tiling and the galaxytargeted strategies.

Past work on optimizing telescope scheduling (Rana et al. 2017) used the tiling method for the purpose of optical followup of GW transients and described three main algorithms for ranking tiles within the GW sky error region in order to generate an observing sequence. They are outlined in brief as follows:

1. Greedy algorithm-ranks tiles in descending order according to probability; does not account for setting tiles.

2. Setting algorithm - selects the highest-probability tiles in each setting window, starting from the earliest setting tiles.

3. Optimal algorithm-reorders the observing sequence generated by the setting algorithm to observe the highest-probability tiles first, without losing setting tiles.

Rana et al. (2017) demonstrate that while the optimal (which is also termed "enhanced") and setting array algorithms have equivalent performance in terms of probability and area coverage within the patch, the optimal array provides an advantage over setting array, as it schedules higher-probability tiles to be observed first. This work builds on the work of Rana et al. (2017) by considering the effects of slew and air mass on the existing algorithms and optimizing over both parameters.

Slew is the process of rotating a telescope to observe different regions of the sky. The slew angle between two tile observations is equivalent to the angular separation between two points on a spherical surface, simply given by the spherical law of cosines as

$$
\begin{aligned}
\sigma_{\text {slew }}= & \cos ^{-1}\left(\sin \left(\mathrm{alt}_{1}\right) \times \sin \left(\mathrm{alt}_{2}\right)\right. \\
& \left.+\cos \left(\mathrm{alt}_{1}\right) \times \cos \left(\mathrm{alt}_{2}\right) \times \cos (\Delta a z)\right) .
\end{aligned}
$$

Here alt $_{1}$, alt 2 , and $\Delta a z=\left|a z_{2}-a z_{1}\right|$ correspond to the two altitude coordinates and the difference between the azimuthal coordinates of each of the points on the sky, assuming an observer-centered horizontal coordinate system. The previous optimization algorithms do not take into account the effect of telescope slewing. Thus, in this paper we demonstrate the effect of slewing in the existing algorithms and propose an alternate algorithm that minimizes the slew between tile observations without loss of tiles or probability.

We also calculate and compare the cumulative air mass among different algorithms for various patch-observatory configurations. Air mass (am) is the length of that part of a light path that lies in Earth's atmosphere; here it is understood that we are referring to the light from the celestial source of interest. Here we employ the expression used by Hardie (1962) 
to estimate the air mass from the zenith angle, $z \equiv 90^{\circ}-$ alt:

$$
\begin{aligned}
\mathrm{am}= & \sec z-0.0018167(\sec z-1) \\
& -0.002875(\sec z-1)^{2}-0.0008083(\sec z-1)^{3}
\end{aligned}
$$

where terms beyond the third order have been dropped. If the zenith angle is not very high, the above air-mass expression can be approximated as am $=\sec z$. This is generally a good approximation, as most observatories set the observing horizon at $25^{\circ}$ or slightly above it. Near the horizon, where light is attenuated by scattering and absorption, the air mass is at its maximum, while at the observatory's zenith, it is unity. However, many of the tiles scheduled to be observed with high air mass will require a long exposure time in order to detect the source, while tiles closer to the zenith could be observed within a brief exposure time. Thus, we implement a modification to the optimal algorithm that will minimize the air mass at which tiles are observed and adjust each tile exposure time based on tile air mass.

In addition to the algorithms presented in Rana et al. (2017), we discuss the following algorithms in this paper:

1. Modified optimal algorithm. This algorithm is listed here as a simplistic reference algorithm against which we compare the slew-optimized algorithm in order to assess its performance. This algorithm modifies the optimal algorithm by removing the low-probability tiles from the end of the sequence that would be lost as a result slewing.

2. Slew-optimization algorithm. We propose a new algorithm to optimize over slew and setting constraints to acquire the maximum probability in the patch.

3. Air-mass-weighted algorithm. We propose another new algorithm to optimize over the air mass and setting constraints to acquire the maximum air-mass-weighted probability in the patch.

All of the algorithms discussed in this paper account for groundbased visibility constraints and only schedule tiles that are above the horizon. We run simulations at the locations of the optical observatories GROWTH-India (GROWTH 2019a, 2019b), Swope (Coulter et al. 2017), and ZTF to systematically compare the performance of the slew optimization and the air-mass-weighted algorithm with that of the modified optimal and greedy algorithms.

\section{Description of Problem}

While previous methods operated under the assumption that the slew time was accounted for in the tile exposure, our calculations demonstrate that the time the telescope spends slewing can cut significantly into the time available for observing tiles in the greedy, setting, or optimal sequence, preventing the telescope from covering all of the tiles provided by the optimal array. Depending on the shape of the patch, the telescope FOV, and the slew rate, the telescope can spend anywhere from tens of minutes to over an hour in slewing to tile patches of a few hundreds of square degrees. In general, this problem of slew times exceeding a tile exposure and resulting in loss of tiles is relevant for telescopes with camera readout times that are less than the average slew time and telescope-patch configurations that require several telescope pointings in order to cover the $95 \%$ credible region. For the rapidly fading transients that optical telescopes seek to follow up, the loss of even a few minutes could make the difference between detection and nondetection of the EM counterpart of a GW source (Rana et al. 2017).

In order to determine how tiles lost to slewing might affect the overall performance of the existing optimal algorithm, we run the optimal algorithm using the FOV, location, and other properties of the GROWTH-India telescope (see Table 1) to tile a $94 \mathrm{deg}^{2}$ patch. First we run the algorithm without accounting for the slew time (original optimal algorithm), and the second time the algorithm calculates the overall time spent slewing and removes the low-probability tiles from the end of the sequence (modified optimal algorithm). We refer to the optimal algorithm with slew accounted for as the modified optimal algorithm. We introduce the modified optimal algorithm to serve as a reference metric against which to compare our slew optimization and to demonstrate the detrimental effect of neglecting to account for slew time within the telescope schedule. If the total time spent slewing exceeds a single or several tile exposures, one or more tiles could be scheduled after they have already set. When we account for slewing, we omit these already-set tiles; as a result, accounting for slew demonstrates that the total probability acquired at the end of the observation is, in reality, lower than predicted by the original code. As demonstrated by Figure 1, in certain cases the original optimal algorithm overestimates the telescope's ability to tile the patch when it does not account for time lost as a result of slewing. The comparison plots of the optimal algorithm tile coverage and cumulative probability are shown in Figures 1 and 2 , respectively.

One of the limiting factors in being able to identify potential transient counterparts to GW events is the ability to resolve faint and distant sources. Depending on the size of the telescope FOV, within a given tile there could be multiple galaxies, each with varying distance to Earth. In order to conduct a less biased search, we choose to adopt a strategy such that the faintest known galaxies within the tile are resolvable. Furthermore, an optical survey is likely to miss even bright sources within tiles that are about to set, as the effects of atmospheric extinction attenuate the light from the source, making it more difficult to observe. For these reasons, when generally conducting optical observations, each target imaged is allotted a different exposure time. Therefore, the tile exposure time, though previously uniformly assigned to all tiles, must change depending on the most distant galaxies within the tile and the tile air mass.

\section{Methods}

\subsection{Slew Optimization}

Past work on slew optimization involved the radio follow-up of blazars (Max-Moerbeck 2014), as the several hours large radio interferometers can spend slewing cuts significantly into the overall source observing time. In the case of radio followup, because the main constraints imposed are the rising and setting times of the patch, optimizing the slew involved applying a slight modification to the well-known "traveling salesman" algorithm (Gutin et al. 2002) to minimize the slew path between the tiles. However, as additional factors such as the telescope FOV, sunrise, and sunset are pertinent for optical follow-up, we cannot simply apply the traveling salesman algorithm on the entire patch to minimize the slew. Instead, we characterize the patches based on the patch visibility, determine 
Table 1

Properties and Parameters of the Optical Observatories Used in Simulations to Test the Slew-optimization and Air-mass-weighted Algorithms

\begin{tabular}{|c|c|c|c|c|c|}
\hline Telescope Name & Location & FOV Size & Latitude & Longitude & $\overline{\text { Altitude }}$ \\
\hline & & $\left(\operatorname{deg}^{2}\right)$ & $(\operatorname{deg})$ & $(\operatorname{deg})$ & (m) \\
\hline GROWTH-India & Ladakh, India & 0.49 & 32.78 & 78.96 & 4500 \\
\hline Swope & Atacama Region, Chile & 3.68 & -29.02 & -70.67 & 2380 \\
\hline ZTF & Mt. Palomar, USA & $7.80^{\mathrm{a}}$ & 33.35 & -116.86 & 1712 \\
\hline
\end{tabular}

Note.

${ }^{\text {a }}$ For our simulations at the location of ZTF, we use the much smaller FOV of its predecessor, PTF.

whether slew optimization will be possible, and then apply the algorithm on them, with the setting and rising constraints imposed. In general, slew optimization will only be possible for cases in which all tiles in a given patch rise above the telescope's horizon. As with the optimal array algorithm (Rana et al. 2017), our starting point is the setting array algorithm. We sketch the process of slew optimization for optical follow-up of GW transients in Figure 3 and describe it in more detail below.

1. Convolve a galaxy catalog with the GW probability distribution on the patch. We modify the tile probability based on the total mass of galaxies contained within each tile, eliminating tiles from the patch that do not contain any galaxies. This step is optional for the slew-optimized algorithm.

2. Run the setting array algorithm (Rana et al. 2017) on the patch. The setting array algorithm provides the set of maximum-probability tiles within the available observation time.

3. Classify patch based on rising/setting. Slew optimization is only possible for certain telescope-patch configurations, so we need to determine whether the patch is rising or setting and whether it will ever rise completely above the horizon.

4. If slew optimization is not possible, run the optimal algorithm on the set of tiles, which would output an observing order for the tiles. (In this case, it would be more advisable to use the air-mass-weighted algorithm).

5. If the patch can be slew optimized, calculate the number of tiles $N$ before first tile sets.

6. Run the traveling salesman algorithm on the first $N$ tiles in the ordered tile array. We only do the slew optimization over these first $N$ tiles so as not to schedule tiles after they have set.

7. Divide the remaining tiles into setting blocks. Here setting blocks are groups of tiles that set at the same time.

8. Run the traveling salesman algorithm on each setting block.

9. Use gained slew time to search for additional tiles. We note here that while in practice we observe that there is almost always some time gained, often the gained time is not sufficient to conduct additional observations. In most cases, checking for gained time yields no additional tiles available for observation, as our method requires tiles to still be above the horizon after all of the tiles selected by the setting array have been scheduled.

10. Repeat steps 4-8 and add to the end of the sequence.
In order to factor the time spent slewing into the observing schedule, we adopt the following procedure. When initially selecting tiles for the optimal sequence, we set the exposure time such that it includes the amount of time it would take for slews less than $20^{\circ}$. The algorithm operates under the assumption that all slews less than $20^{\circ}$ will take the same amount of time as a $20^{\circ}$ slew, as it is difficult for telescopes to maintain exact slew times for small slew angles $\left(\sigma_{\text {slew }} \leqslant 20\right)$, due to the acceleration and deceleration of the telescope. Therefore, we allot a time gap such that $t_{\text {gap }}=t_{\text {expo }}+T_{20}$, where $T_{20}$ is the approximate time taken for slews less than $20^{\circ}$ and $t_{\text {expo }}$ is the desired tile exposure time. Here $T_{20}=(20$ $\operatorname{deg}) /\left(v_{\text {slew }}\right)$, where $v_{\text {slew }}$ is the telescope slew rate.

Once we have run the traveling salesman algorithm on our set of tiles, we shift the observation times by $\max$ (readout time, exact slew time). For cases in which slews can be completed within the telescope readout time, we need only shift the observation time by the readout time.

In step 9, we determine whether we can observe additional tiles by calculating the gained time. We define the gained time as follows:

$$
\begin{aligned}
t_{\text {gained }}= & \left(20 / v_{\text {slew }}\right) * N_{\text {tiles }}-t_{\text {slew,exact }} \\
& +\left((T>20)_{\text {optimal }}-(T>20)_{\text {slew }}\right),
\end{aligned}
$$

where the first term is the difference between the accounted-for slew time and the exact amount of time spent slewing and the second term records the difference between time spent performing slews greater than $20^{\circ}$ before and after slew optimization.

Although the traveling salesman algorithm can be shown to be the optimal solution for minimizing the distance between two points, due to the additional constraints imposed by optical follow-up, our slew-optimization algorithm, unlike the airmass-optimization algorithm, is not a unique or completely optimized solution. We prescribe the slew-optimization algorithm as a heuristic method, and as we will show in Section 4, we find in simulations that it shows significant improvement in overall slew angle acquired.

\subsection{Air Mass and Exposure Time}

Our objective is to maximize the total probability coverage based on the setting time and the air mass of the tiles on the GW localization. The tiles on the localization move as Earth rotates, and the air mass of the tiles changes as the altitude of the tiles changes with time. The air mass of a given tile changes at each observation time. Therefore, we propose an algorithm to maximize the total probability coverage over the setting and 



Figure 1. Tile coverage on a $94 \mathrm{deg}^{2}$ sky patch with a $0.5 \mathrm{deg}^{2}$ FOV at the location of the GROWTH-India telescope (Ladakh, IN) using the optimal algorithm, without accounting for slew (left) and the modified optimal algorithm (right). We assume a tile exposure time of $300 \mathrm{~s}$, based on the current estimates for the exposure time needed to observe a 20th magnitude source (GROWTH 2019b). The telescope has a slew rate of $2 \mathrm{deg} \mathrm{s}^{-1}$. Each tile, indicating an image taken by the telescope, is represented using a black square; untiled regions remain reddish, the color of the patch. The original algorithm schedules the sample observatory to 186 of the 197 total tiles; by accounting for slew, the optimal schedule can only cover 114, a loss of 62 tiles from the original 186 it was scheduled to cover. As is demonstrated above, in this telescope-patch configuration, the original optimal algorithm (Rana et al. 2017), which does not account for slewing, overestimates the telescope's ability to tile the patch.

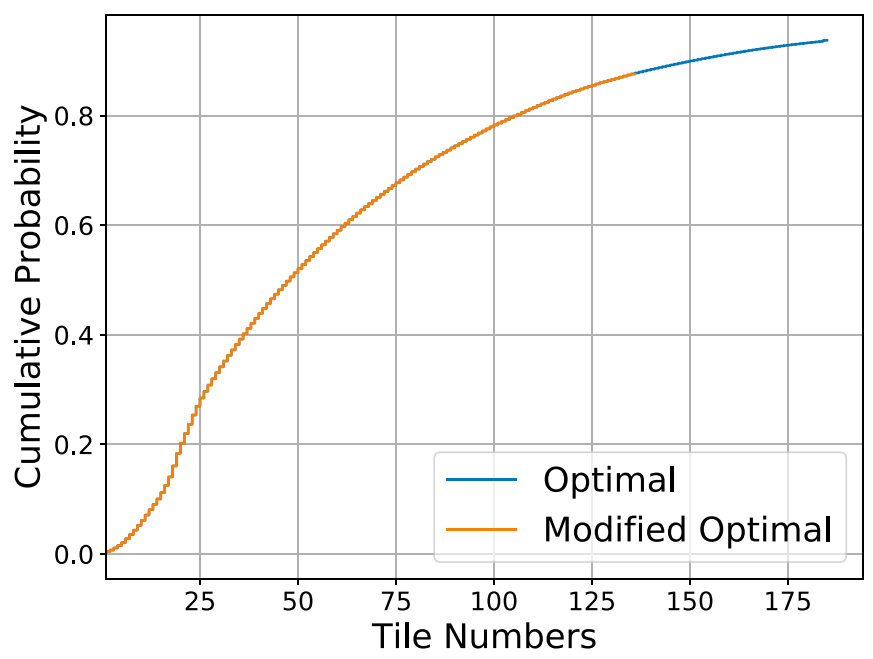

Figure 2. Cumulative probability on a $94 \mathrm{deg}^{2}$ sky patch using the optimal algorithm, without accounting for slew (blue curve) and the modified optimal algorithm (orange curve). The telescope has a slew rate of $2 \mathrm{deg} \mathrm{s}^{-1}$. The plots chart the probability covered within the patch as a function of the number of images taken. The original algorithm predicts that the sample observatory can cover a probability of $93.7 \%$; accounting for slew, the observatory is only able to cover $82.1 \%$ probability. As is demonstrated above, the amount of probability acquired for this telescope-patch configuration after accounting for slew is much less than predicted by the original optimal algorithm (Rana et al. 2017).

air mass of the GW localization. We use the Hungarian optimization algorithm (Munkres 1957) to get the optimal solution for this problem. In our algorithm, we define a new probability called the air-mass-weighted probability. The airmass-weighted probability of a tile is the ratio between the galaxy-gw probability and the air-mass weight of the tile, defined in Equation (6). Although the galaxy-gw convolved (or GW) probability is fixed at all times for each tile in the localization, the air-mass-weighted probability of each tile varies with time. We determine the air-mass-weighted probability for each tile at all different times, using the timedependent air mass, and use our algorithm to select the schedule of tiles that will maximize the air-mass-weighted probability. We demonstrate the algorithm pointwise below:

1. Convolve the GW probability with a galaxy catalog. We modify the GW probability based on the total mass of galaxies contained in the given localization. We make a grid of tiles based on the convolved probability, eliminating those tiles that do not contain any galaxies. This is an optional step in our algorithm.
2. Account for air mass. We approximated the air mass in the following way to schedule the observation: ${ }^{4}$

$$
\operatorname{am}(z)=\left\{\begin{array}{ll}
\frac{1}{\cos (z)}, & \text { if } z \leqslant 90^{\circ}-\operatorname{alt}(\text { horizon) } \\
\infty, & \text { if } z>90^{\circ}-\operatorname{alt}(\text { horizon) }
\end{array},\right.
$$

where $\operatorname{am}(z)$ is the air mass as a function of time, $z$ is the zenith angle in degrees as defined before as a function of time, and alt(horizon) is the altitude of the observing horizon of the telescope. The apparent magnitude $m_{\lambda}(z)$ at wavelength $\lambda$ of a source at zenith angle $z$ for a groundbased telescope can be expressed as

$$
m_{\lambda}(z)=m_{\lambda}+C_{\lambda} \operatorname{am}(z),
$$

where $m_{\lambda}$ is the apparent magnitude of the source measured outside of Earth's atmosphere. $C_{\lambda}$ is a constant, depends on the wavelength, and varies from observatory to observatory. The apparent magnitude of the source toward the zenith becomes $m_{\lambda}\left(z=0^{\circ}\right)=m_{\lambda}+C_{\lambda}$. The exposure time to observe a source of fixed $m_{\lambda}$ at a fixed signal-to-noise ratio and at an air mass $\operatorname{am}(z)$ by a ground-based telescope can be expressed as

$$
\begin{aligned}
& t_{\exp }=t_{\exp }\left(z=0^{\circ}\right) \times 10^{0.4 C_{\lambda}(\operatorname{am}(z)-1)}, \\
& t_{\exp }=t_{\exp }\left(z=0^{\circ}\right) \times w_{\mathrm{am}},
\end{aligned}
$$

where we calculate an air-mass weight $w_{\mathrm{am}}=$ $10^{0.4 C_{\lambda}(\mathrm{am}(z)-1)}$ for a tile such that we can account for the change in the exposure time. We note that different observatories may choose to weight the two factors, $p_{\mathrm{gw}}$ and $w_{\text {am }}$, differently. The air-mass-weighted probability of a tile is inversely proportional to the $w_{\mathrm{am}}$ :

$$
p_{\mathrm{amw}}=\operatorname{Norm}\left[\frac{p_{\mathrm{gw}}}{w_{\mathrm{am}}}\right],
$$

where $p_{\mathrm{gw}}$ is the GW (or galaxy-gw) probability, $p_{\mathrm{amw}}$ is the air-mass-weighted probability, and Norm is the normalization constant chosen such that the total air-mass-weighted probability is unity. Our strategy here is to devote less telescope time to tiles at higher air mass, so that low-airmass, high-probability tiles are prioritized for observation. At any given time, if the altitude of a tile becomes less than the observing horizon, we make the air-mass value infinity so that the air-mass-weighted probability will become zero. Using Equations (1) and (6), we account for the setting and rising of all the tiles in the localization.

\footnotetext{
This is a good approximation of the air mass when alt(horizon) $\sim 25^{\circ}$.
} 


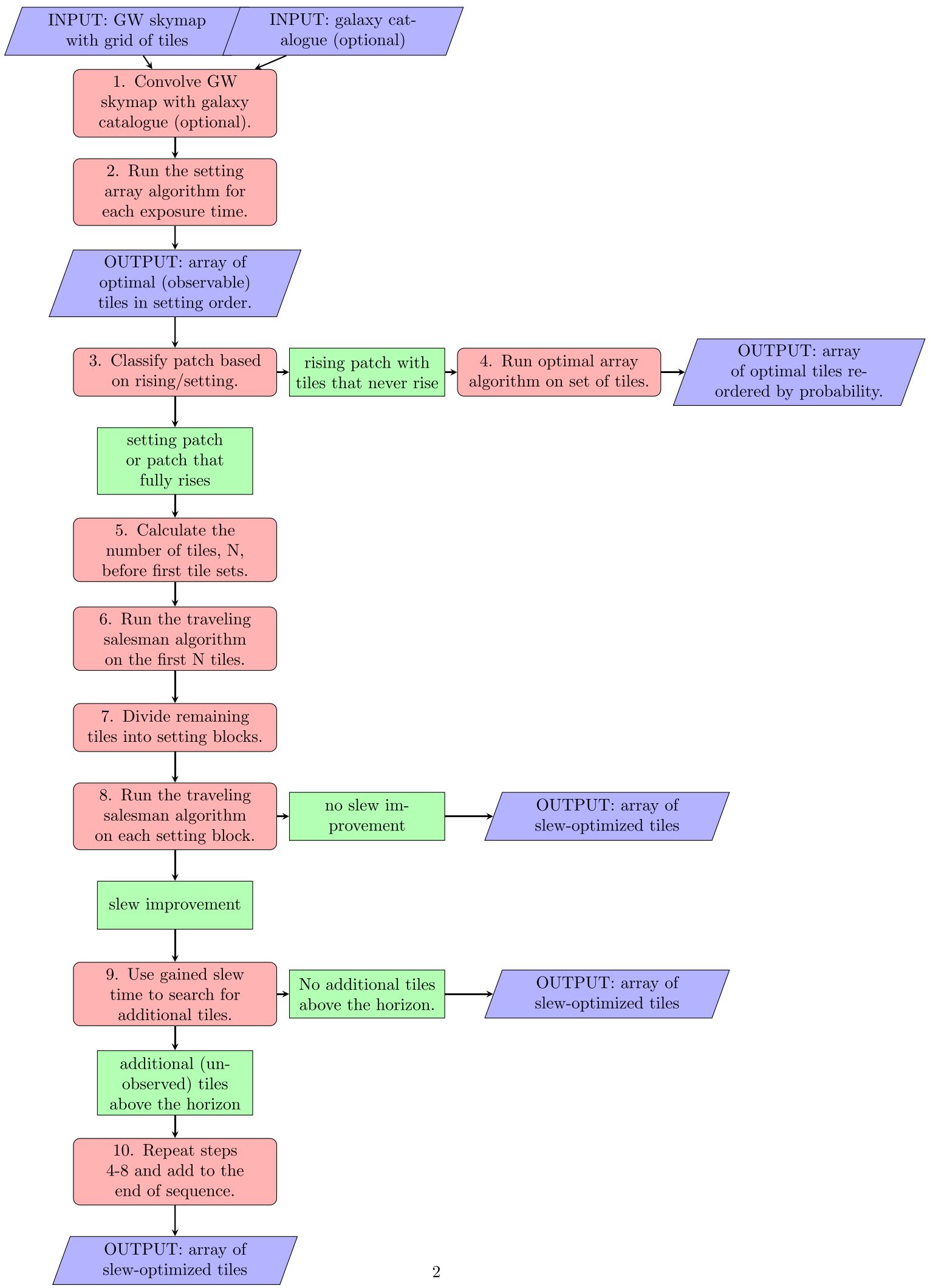

Figure 3. Flowchart diagrammatically illustrating the various steps that go into the slew-optimization algorithm. The parallelograms correspond to inputs and outputs, rectangular boxes correspond to decisions made, and the rounded-edge boxes outline the processes.

To illustrate how the tile air mass affects $p_{\text {amw }}$, we can consider an example where two tiles with the same galaxygw probability are at different altitudes. If one tile is at an altitude of $30^{\circ}$ from the horizon and the other is located at the zenith, the first tile will have double the air mass of the tile at the zenith, so the air-mass-weighted probability of that tile will be half of the air-mass-weighted probability of the tile at the zenith. 
We calculate $p_{\text {amw }}$ for every tile for all time steps from the observation start time to the observation end time. We make a table, where the rows represent the time steps (one time step is equivalent to one exposure) and the columns represent the tiles in the localization. One element in the $i$ th row and $j$ th column is the air-mass-weighted probability of finding the source at the $j$ th tile at the $i$ th time step.

3 . Use the Hungarian algorithm to maximize the air-massweighted probability. If more than one optimal solution exists, we choose the solution where higher-probability tiles with lower air mass are scheduled earlier. More than one optimal solution exists when a few tiles can be observed in multiple time windows without changing the total $p_{\text {awm. }}$. In that case we choose a tile at that time window where air mass is lowest. To demonstrate that, we consider an example of two tiles $\mathrm{tl}_{1}$ and $\mathrm{tl}_{2}$ with $\mathrm{GW}$ probability $p_{\mathrm{gw} 1}$ and $p_{\mathrm{gw} 2}$ respectively, where $p_{\mathrm{gw} 1}$ is greater than $p_{\mathrm{gw} 2}$. The tile $\mathrm{tl}_{1}$ can be observed in two air masses $\mathrm{am}_{1}$ and $a m_{1}^{\prime}$, where $\mathrm{am}_{1}>\mathrm{am}_{1}^{\prime}$. Another tile $\mathrm{tl}_{2}$ can also be observed in two air masses $\mathrm{am}_{2}$ and $\mathrm{am}_{2}^{\prime}$, where $\mathrm{am}_{2}<\mathrm{am}_{2}^{\prime}$. In this situation one optimal solution is the $\mathrm{tl}_{1}$ with air mass $\mathrm{am}_{1}$ and $\mathrm{tl}_{2}$ with air mass $\mathrm{am}_{2}$. The other possible solution without breaking the optimality is scheduling $\mathrm{tl}_{1}$ at air mass $\mathrm{am}_{1}^{\prime}$ and $\mathrm{tl}_{2}$ at air mass $\mathrm{am}_{2}^{\prime}$. In the air-mass-weighted method, we choose $\mathrm{tl}_{1}$ with air mass $a_{1}$ earlier than $t_{2}$ with air mass $a_{2}$.

4. Allocate exposure time. For a galaxy-targeted hunt, we adjust each tile exposure time based on the most distant galaxy within that tile, as the source's flux decreases as its distance squared:

$$
t_{\exp }=\frac{d_{\mathrm{gal}}^{2}}{d_{0}^{2}} t_{\exp 0},
$$

where $t_{\exp }$ is the adjusted exposure time of the tile, $t_{\exp 0}$ is the starting exposure time assigned to all tiles, $d_{0}$ is the maximum distance within the 3D GW localization within the tile, and $d_{\text {gal }}$ is the distance to the farthest galaxy in the tile. If the telescope FOV is large enough, all tiles will contain both nearby and distant galaxies such that the required exposure time to resolve distant galaxies in each tile will average out. We impose a maximum distance based on either the maximum distance within a tile in the 3D GW localization or the telescope sensitivity such that galaxies more distant than this maximum distance are not considered when we allocate exposure time.

Then, we apply a second adjustment to the tile exposure time according to Equation (5) based on its air mass. In allocating our exposure time, we do not account for the fading light curve of the counterpart.

The starting exposure time is a user-defined input into our code. One should set the starting exposure time by first selecting one tile in the localization and then determining the amount of exposure time needed in order to observe a source at some desired magnitude out to the maximum distance within the tile (based on the 3D GW localization). Our code assigns that input exposure time as the initial exposure time for all tiles and then applies the adjustments described above. In our simulation, we use the highest-probability tile within the localization to set our starting exposure time.
Combining the air-mass-weighted method with the slewoptimization method is very complicated, as the air-massweighted method breaks the order determined by the slewoptimization algorithm that will minimize the slew between tiles. For this reason, we do not attempt to combine the slew and air-mass optimizations. The solution to this problem is still being formulated and is outside the scope of the current work. Instead, we show our results for the air-mass-weighted algorithm separately from the slew-optimization method, for which we only adjust the exposure times of the tiles appropriately in the finalized sequence. In this section, the two new methods we have presented are to be considered as alternative methods by which to schedule observations.

The question of which algorithm to use in a given situation now becomes relevant. The most direct way to determine which scheduling method is most suitable for a given telescope-patch configuration is to run both algorithms on the patch and choose the algorithm that covers the most GW probability. On a standard $2.4 \mathrm{GHz}$ processor, the slewoptimization algorithm takes $\sim 3-5$ minutes to complete and the air-mass-weighted method takes less than 1 minute to complete, so running both algorithms before the start of the observation does not present any significant overheads in time. The cumulative GW probability acquired is directly comparable between both algorithms, as the GW probability relies only on the GW sky map to inform about the true location of the source.

For the galaxy-targeted search, an additional complication is the incompleteness of galaxy catalogs such as CLU and Glade (Cook et al. 2017; Dálya et al. 2018) out to distances larger than $\sim 200 \mathrm{Mpc}$. However, since most optical telescopes are only sensitive out to about $200 \mathrm{Mpc}$, this should not significantly bias the results.

\section{Results}

In this section, we use both case studies and simulations to test the performance of our two algorithms. For the purpose of this study, the telescopes we select (GROWTH-India, Swope, and ZTF) are optical telescopes apt for rapid imaging and follow-up of transient events. For our different simulations, we vary their FOV sizes from 0.5 to $8 \mathrm{deg}^{2}$. Table 1 shows the locations and parameters of each of the telescopes used.

We revisit the example referred to earlier in Section 2 to see how the slew-optimization algorithm performance differs from that of the modified optimal algorithm for the GROWTH-India telescope. Instead of comparing the performance of the slewoptimization algorithm with the original optimal algorithm that usually overestimates the available time for observation, we choose to compare the performance of the slew-optimization algorithm against the heuristic modified optimal algorithm. We can compare the cumulative probability in each case using Figure 4. Without taking slewing time into account, the telescope is capable of covering all of the tiles in the patch, covering a cumulative probability of $93.7 \%$ (see Figure 1). On the other hand, the modified optimal algorithm can only cover a probability of $82.1 \%$. The slew-optimization algorithm is designed such that it cannot perform worse than the modified optimal algorithm, in terms of probability and coverage; for the cases in which the slew cannot be further optimized, the algorithm will return the same tile schedule as the modified optimal algorithm. In this case, the slew-optimization algorithm acquires about the same amount of probability $(82.1 \%)$ as the 


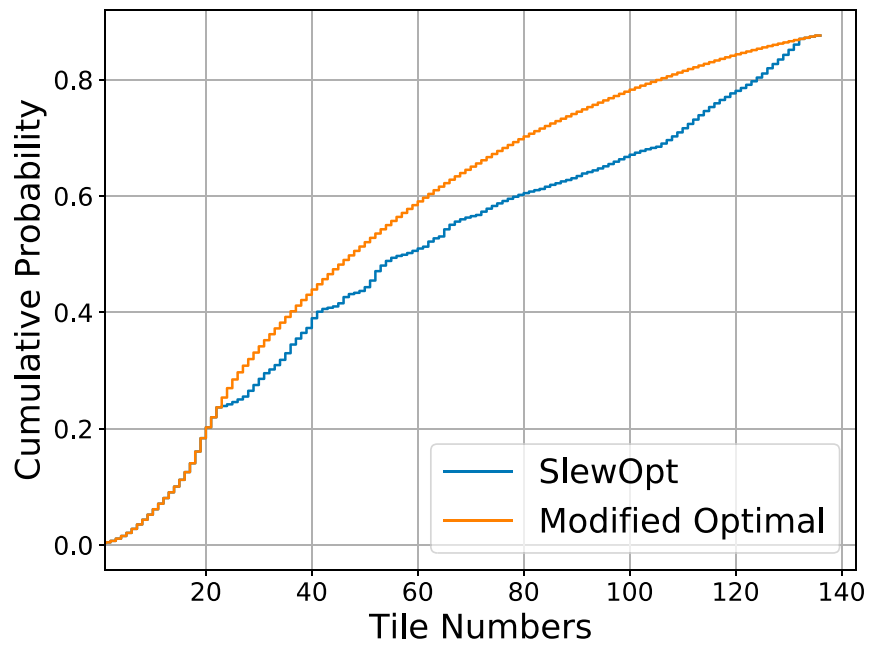

Figure 4. Cumulative probability on a $94 \mathrm{deg}^{2}$ sky patch using the modified optimal algorithm (left) and slew-optimization algorithm (right). The telescope has a slew rate of $2 \mathrm{deg} \mathrm{s}^{-1}$. The plots chart the probability covered within the patch as a function of the number of images taken. The slew-optimization algorithm acquires about the same amount of probability as the modified optimal algorithm (see Figure 2).

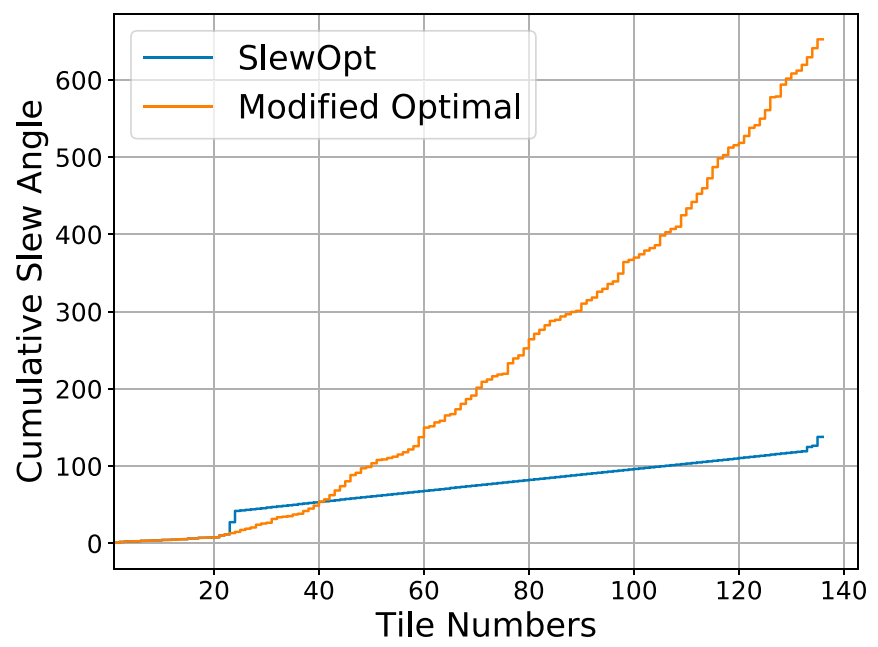

Figure 5. Cumulative slew angle on a $94 \mathrm{deg}^{2}$ sky patch using the modified optimal algorithm (orange curve) and slew-optimization algorithm (blue curve). The telescope has a slew rate of $2 \mathrm{deg} \mathrm{s}^{-1}$. The plots chart the telescope's cumulative slew angle over its observation of the patch. For the slewoptimization algorithm, the cumulative slew angle increases steadily, with two slew jumps around tiles 25 and 130. After optimizing for slew, the telescope's cumulative slew angle reduces by about $300^{\circ}$, allowing additional time for further observation.

modified optimal algorithm, though both algorithms far surpass the amount of probability covered by the greedy algorithm. The key advantage of the slew-optimized algorithm is demonstrated in Figure 5 by the cumulative slew angle being reduced from over $600^{\circ}$ to about $140^{\circ}$ as a result of slew optimization. The time saved in slewing aids in more rapid observation of the patch, which is important especially when there are multiple targets to observe in one night.

By comparing cumulative slew angles between the slewoptimized array and the modified optimal array, we can see that the curve shapes differ quite drastically. The optimal method schedules tiles to be observed with small slew jumps between observations, resulting in a relatively smooth increase in overall slew angle with tile number. On the other hand, the slew-
Table 2

Telescope Performance with Varying Slew Rate

\begin{tabular}{lcccc}
\hline \hline$v_{\text {slew }}\left(\mathrm{deg} \mathrm{s}^{-1}\right)$ & Algorithm & Prob. & $\sigma_{\text {slew }}(\mathrm{deg})$ & $t_{\text {slew }}($ minutes $)$ \\
\hline 2 & optimal & 0.683 & 2459 & 23.42 \\
\hline 5 & slewopt & 0.714 & 2095 & 17.47 \\
\hline optimal & 0.728 & 3099 & 10.33 \\
\hline 10 & slewopt & 0.730 & 2501 & 8.33 \\
\hline & optimal & 0.737 & 3283 & 5.47 \\
\hline
\end{tabular}

Note. The table above displays the difference between the performance of the optimal and slew-optimization algorithms with slew rates $\left(v_{\text {slew }}\right)$ of 2, 5, and 10 $\operatorname{deg~s} \mathrm{s}^{-1}$ in terms of cumulative probability, slew angle in degrees, and slew time $\left(t_{\text {slew }}\right)$ in minutes. The slew times here have already been factored into the tile observation times. The sample observatory is at the location of Swope, with a $1 \mathrm{deg}^{2}$ FOV. With increasing slew rate, the cumulative probability and slew angle increase for cases in which the telescope cannot tile the entire $95 \%$ credible region of the sky map, while the slew time always decreases. As indicated by the increase in probability for increasing slew rate, this is because the faster-slewing telescopes have the ability to tile more of the patch before it sets. As expected, slew optimization is more critical for telescopes with slower slew rates.

optimized method is mostly linear with two jumps around the 25th and 130th tiles in the schedule. In effect, the slewoptimization method attempts to schedule as many tiles consecutively as possible before jumping to a different region of the patch. Figure 5 displays the behavior of the slewoptimization algorithm as expected.

The telescope slew rate also influences the amount of improvement the slew-optimization algorithm has over the modified optimal algorithm. Table 2 indicates that slew optimization is vital for slowly slewing telescopes that are more likely only to cover a small portion of the patch within the time constraint owing to time spent slewing. For cases in which the telescope cannot tile the entire patch within the time constraint, faster-slewing telescopes tend to have a larger cumulative slew angle at the end of the slew-optimized schedule because they can acquire a larger amount of probability than slowly slewing telescopes. Table 2 shows that even for telescopes with fast slew rates of $10 \mathrm{deg} \mathrm{s}^{-1}$, slew optimization can reduce the overall time the telescope spends slewing.

In a similar fashion we check how the telescope FOV influences the performance of slew optimization compared to modified optimal. We vary the FOV of the telescope, assuming a slew rate of $2 \mathrm{deg} \mathrm{s}^{-1}$ and fixing the location and other parameters to that of Swope's. Table 3 demonstrates the relative improvement of the slew-optimization algorithm over the modified optimal algorithm; we note that there is more improvement for smaller FOV sizes. Therefore, the problem of slew optimization is especially relevant for smaller-FOV telescopes with slow slew rates attempting to tile large $\mathrm{GW}$ sky error regions.

Using a different set of patch and telescope parameters, we now perform a comparison between the air-mass-weighted and greedy algorithms, observing a marked improvement when using the air-mass-weighted algorithm. Figure 6 shows the cumulative probability coverage by the ZTF telescope (with an $8 \mathrm{deg}^{2}$ FOV) with the greedy and air-mass-weighted methods for a given patch. Around tile 35 the optimal algorithm deviates 
Table 3

Telescope Performance with Varying FOV

\begin{tabular}{lcccc}
\hline \hline FOV $\left(\mathrm{deg}^{2}\right)$ & Algorithm & Prob. & $\sigma_{\text {slew }}(\mathrm{deg})$ & $t_{\text {slew }}($ minutes $)$ \\
\hline 1.0 & optimal & 0.706 & 2810 & 23.4 \\
\hline 2.0 & slewopt & 0.714 & 2095 & 17.5 \\
\hline & optimal & 0.888 & 4891 & 32.5 \\
\hline 3.0 & slewopt & 0.890 & 4399 & 36.7 \\
\hline & optimal & 0.945 & 3899 & 40.8 \\
\hline
\end{tabular}

Note. The table above displays the difference between the performance of the optimal and slew-optimization algorithms with telescope FOVs of 1.0, 2.0, and $3.0 \mathrm{deg}^{2}$ in terms of cumulative probability, slew angle in degrees, and slew time in minutes. The slew times here have already been factored into the tile observation times. The sample observatory is at the location of Swope, with a slew rate of $2 \mathrm{deg} \mathrm{s}^{-1}$. For this case, in which the smaller-FOV instruments are unable to tile the entire localization region, we see that with increasing FOV the overall slew angle increases since the larger-FOV instruments cover more overall area in the patch. If both a large- and small-FOV instrument could tile the entire patch, the larger-FOV instrument would have a lower cumulative slew angle in the schedule. In general, we find that slew optimization is more important for smaller-FOV telescopes.



Figure 6. Cumulative probability as a function of tile number at the location of the ZTF observatory using the air-mass-weighted algorithm. The plots chart the probability covered within the patch as a function of the number of images taken. The air-mass-weighted algorithm (blue curve) covers a probability of $36.5 \%$, while the greedy algorithm (orange curve) covers a probability of $32.8 \%$. When comparing the performance of this modified optimal algorithm with the greedy algorithm, we observe a marked improvement in probability gain.

from greedy, appearing to acquire probability more gradually at first, but eventually surpassing the greedy algorithm in tiles and probability covered. The greedy method and air-mass-weighted method cover $32.8 \%$ and $36.5 \%$ probability, respectively. We do not account for air mass in the greedy method. When comparing Figure 6 to the cumulative probability acquired by the original optimal algorithm, we observe that the curve corresponding to the optimal algorithm has the same shape as the air-mass-weighted algorithm but deviates from the greedy curve earlier in tile number. This indicates that the air-mass-weighted algorithm

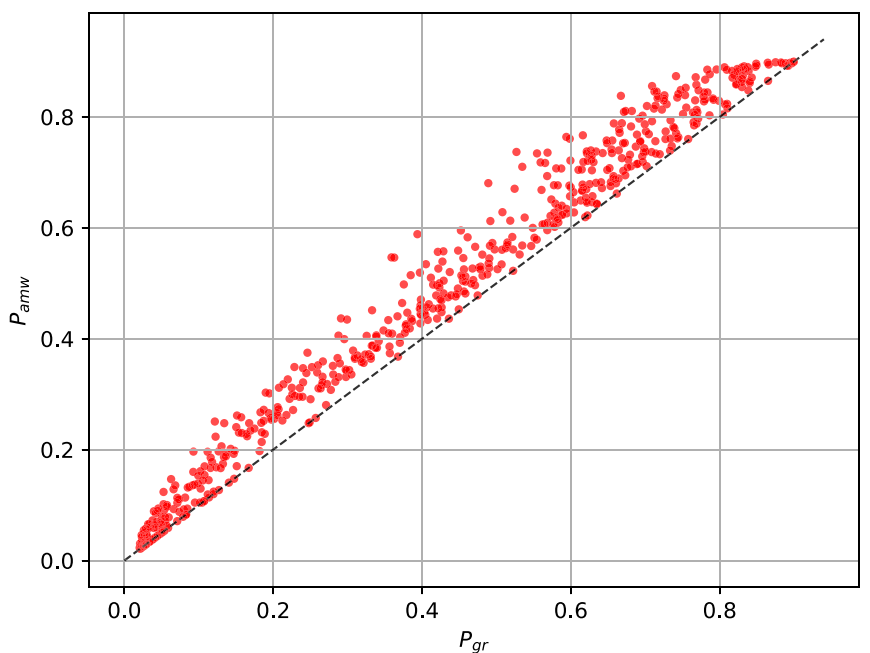

Figure 7. Comparison between the air-mass-weighted method and the greedy method for 616 localizations. The $x$-axis is the probability covered by the greedy method $\left(P_{\mathrm{gr}}\right)$. The $y$-axis is the probability covered by the air-massweighted method. The dashed line represents equal coverage by both the methods. Most of the points lie above the dashed line, indicating that $P_{\mathrm{amw}}>P_{\mathrm{gr}}$ for nearly all of the simulated localizations.

prioritizes observing higher-probability tiles earlier on in the observing schedule.

In order to test the performance of our air-mass-weighted algorithm, we generate a distribution of GW sky localizations for BNSs, assuming that the sources are distributed uniformly in volume. As our study pertains to the ADE, in which we expect our detectors to have improved in sensitivity over the current state of GW detectors, the sky patches we use are distributed between areas of 15 and $225 \mathrm{deg}^{2}$. Though our algorithm can be run with various different input parameters for the FOV, coordinates, altitudes, and other observatory-specific parameters, we choose the location and parameters of the GROWTH-India telescope, changing the original FOV to a $1^{\circ} \times 2^{\circ}$ square FOV to determine in which cases the air-massweighted algorithm shows an improvement over the greedy algorithm. For our simulations with the GROWTH-India telescope, we keep the observing horizon at an altitude of $30^{\circ}$ (air mass $\sim 2$ ), and the observations are performed when the Sun is below $-12^{\circ}$ altitude. We use $C_{\lambda}=0.1$ for the simulation. In our code, however, one can set the horizon as is appropriate for the follow-up telescope, because GW localizations can be arc shaped and extended, and in some cases telescopes may wish to observe a source even below $25^{\circ}$, which will increase the duration of the observation. We assume that the luminosity of the optical counterpart does not change with time, which fixes an exposure time of $300 \mathrm{~s}$ to see a source at a distance of $100 \mathrm{Mpc}$. The exposure time changes for different localizations based on their average distance. We ran the air-mass-weighted and greedy methods on 900 of these 3D localizations for the GROWTH-India optical telescope. Out of these, 616 were visible from the GROWTH-India observatory; the localizations at the far south were not visible.

Figure 7 presents a scatter plot of the covered total probability by the air-mass-weighted and greedy methods. The $x$-axis and $y$-axis display the probability covered by the greedy algorithm $\left(P_{\mathrm{gr}}\right)$ and the probability covered by the airmass-weighted algorithm $\left(P_{\mathrm{amw}}\right)$, respectively. The dashed line 


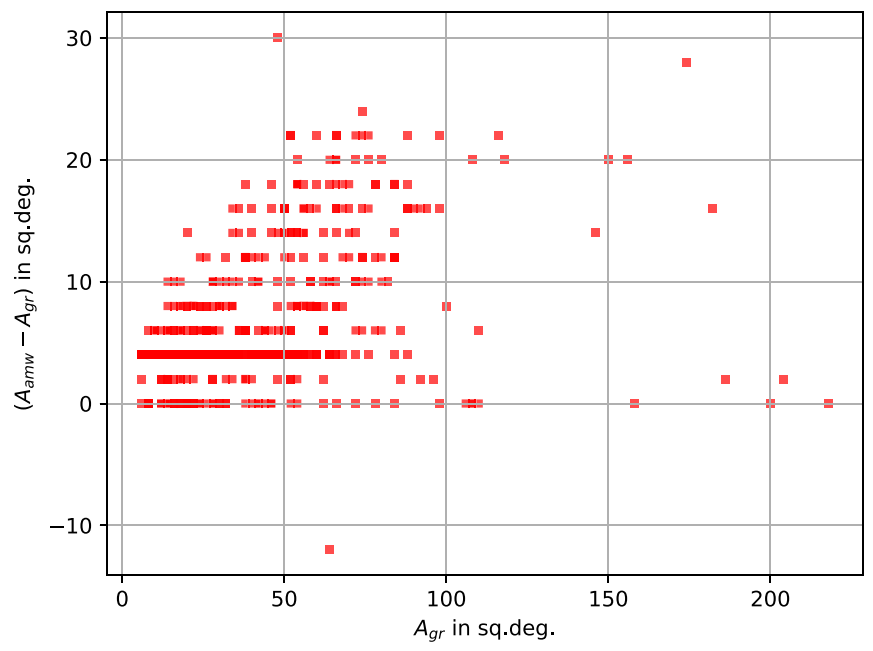

Figure 8. Comparison between the areal coverage for the air-mass-weighted method and the greedy method. The $x$-axis is the area covered by the greedy algorithm $\left(A_{\mathrm{gr}}\right)$. The $y$-axis is the difference between the area covered by the air-mass-weighted algorithm and the greedy algorithm $\left(A_{\mathrm{amw}}-A_{\mathrm{gr}}\right)$. Note that one data point lies below zero, indicating that in one case the air-massweighted method resulted in a loss of areal coverage. Because the greedy method does not account for setting or air mass, it often misses the highprobability setting tiles and uses the available time to schedule low-probability tiles. While the air-mass-weighted method always acquires more GW probability than the greedy method, sometimes it misses low-probability tiles scheduled by the greedy method, resulting in a loss of areal coverage.

represents equal coverage by both of the methods. Most of the points fall above the dashed line, indicating that in most cases $P_{\text {amw }}>P_{\text {gr }}$ regardless of the amount of probability acquired by the greedy method. In Figure 8 , we compare the total areal coverage by the air-mass-weighted method with that by the greedy method. Here the $x$-axis is area covered by the greedy algorithm, $\left(A_{\mathrm{gr}}\right)$, and the $y$-axis is the difference between the area covered by the air-mass-weighted algorithm and the greedy algorithm, $\left(A_{\mathrm{amw}}-A_{\mathrm{gr}}\right)$. Each additional tile acquired by the air-mass-weighted method over greedy adds an area of 2 $\mathrm{deg}^{2}$ because of the $2 \mathrm{deg}^{2}$ FOV we use for these simulations. The general trend we observe from Figures 7 and 8 is that the improvement in areal or probability coverage between the airmass-weighted and greedy algorithms increases with increasing $P_{\mathrm{gr}}$ or $A_{\mathrm{gr}}$. This is because when patches are visible for a longer duration of time, both algorithms can cover a larger area of the patch, and the air-mass-weighted algorithm can determine a more optimal solution to maximize the air-mass-weighted probability.

To demonstrate the relative improvement in the total probability coverage between the two methods, we show Figure 9, in which the $x$-axis represents the probability covered by the greedy algorithm, $P_{\mathrm{gr}}$, while the $y$-axis represents the difference between the probabilities covered by the air-mass-weighted method and the greedy method, expressed as a percentage of the greedy probability coverage $\left(\left(P_{\mathrm{amw}}-P_{\mathrm{gr}}\right) / P_{\mathrm{gr}}\right)$. When the probability covered by the greedy method is less than $\sim 10 \%$ of the total GW probability in a sky patch, we see that the air-mass-weighted method can cover more than double the probability acquired by the greedy method in the same patch. However, even when the greedy method covers a large percentage $(\sim 80 \%)$ of the GW localization, the air-mass-weighted method is capable of covering up to $10 \%$ more probability than the greedy method, which is consistent with the earlier trend we observed. In short, our results

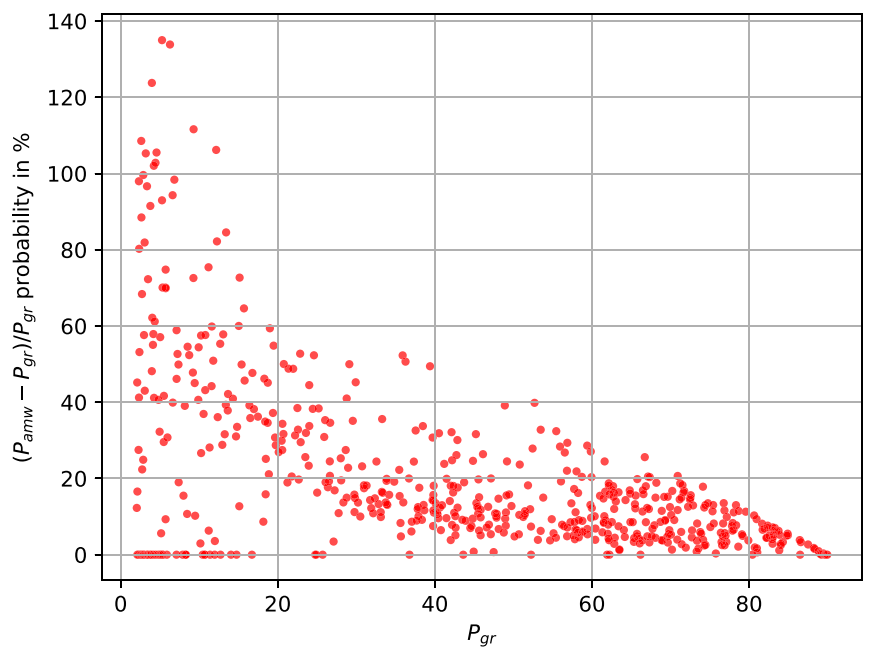

Figure 9. Relative improvement in total probability coverage by the air-massweighted and greedy methods. The $x$-axis is the GW probability covered by the greedy algorithm, $P_{\mathrm{gr}}$, in percentage. The $y$-axis is the difference between the probability covered by the air-mass-weighted method and the greedy method as a function of greedy probability coverage, $\left(P_{\mathrm{amw}}-P_{\mathrm{gr}}\right) / P_{\mathrm{gr}}$, in percentage. Note that most of the points are lying above zero, which implies that the airmass-weighted method performs better than the greedy method.

from simulations run to test the air-mass-weighted method are a strong indication of the robustness of the algorithm.

\section{Discussion}

\subsection{Summary}

The optimal algorithm presented in Rana et al. (2017) provides an optimized telescope scheduling method for observing large sky error regions that accounts for several observational constraints, including the setting and rising of tiles, Sun, Moon, and telescope time constraints. In this paper, we demonstrate the need to improve this algorithm by optimizing over slew and air mass, as the time spent slewing and additional exposure time required to resolve high air-mass tiles detract from the overall time available for imaging the patch. We present two algorithms-air-mass-weighted and slew-optimized-and describe criteria for choosing which algorithm to use to schedule observations. One important modification we make to the original optimal algorithm is adding in an optional step in both algorithms to convolve the GW patch with a galaxy catalog, combining the tiling and galaxy-targeted search strategies. We demonstrate that both algorithms presented in this work are more realistic than the original optimal algorithm and improve over the greedy algorithm not only in terms of cumulative air mass and slew but also in terms of overall probability acquired.

\subsection{Caveats}

Many of the caveats of the original optimal algorithm remain true for our newly proposed algorithms, as do the procedures for overcoming these caveats. We briefly summarize these caveats below (see Section 4.2 of Rana et al. 2017 for a more detailed discussion).

We assume that the source light curve stays flat throughout the duration of the observation. GW170817 is the only BNS merger event observed so far, and its optical counterpart was first seen $\sim 11 \mathrm{hr}$ after the merger. Therefore, we do not yet have any observational information about the evolution of the 
light curve during the $11 \mathrm{hr}$ immediately following the merger. Theoretically, the optical and infrared light curves of the counterpart of the BNS merger might vary depending on the neutron star masses and equation of state. Similarly, no NSBH mergers have been observed yet. Consequently, we have chosen to be agnostic about this aspect and opted for a flat light curve here. Future studies may extend this work based on any new information that arises from anticipated merger observations involving neutron stars in the coming years.

We do not account for partial cloud cover in our scheduling since it is outside the scope of this work. This could be a potential weakness in most telescope scheduling algorithmsthe partial cloud cover constraints should be addressed in a future work.

As mentioned in the previous work, synoptic surveys will often image based on a predefined grid for comparison of previously imaged fields; again, here offsetting our grid tiles (placed based on the maximum GW probability) to match the predefined grid should not significantly affect the performance of either algorithm.

The true tile air mass, though parameterized as purely a function of the tile altitude, depends on atmospheric visibility and the color of filter used to conduct the observation. We do not account for "seeing" or filters in our air-mass calculation, as it would make our algorithm very telescope specific; instead, we use Equation (1) given in Section 3.2 to calculate the air mass of the tiles.

As proposed in Rana et al. (2017), one can adjust the final tile observation time to account for counterparts that may fade on timescales shorter than a day. We anticipate, however, that in most cases optical observatories tiling GW or GRB localization patches will continue observation until there is confirmed nondetection. As evidenced by the first joint GW-EM detection in 2017, it will be difficult to determine exactly the timescale on which an optical counterpart will fade. However, the air-massweighted algorithm addresses this in part by allocating longer exposure times to tiles containing more distant galaxies.

This work made use of the Python libraries Numpy and Matplotlib. It also made use of Astropy, a communitydeveloped core Python package for Astronomy (Astropy Collaboration et al. 2013, http://www.astropy.org). We would like to thank Patrick Brady, Leo Singer, Varun Bhalerao, G. C. Anupama, Om Sharan Salafia, and Shaon Ghosh for helpful discussions. We would like to especially acknowledge Varun Bhalerao for providing us with with both broad and detailed comments and feedback on our manuscript. We would also like to thank Michael Coughlin for carefully reading the manuscript and making useful comments in LIGO P\&P review (https://dcc.ligo.org/LIGO-P1900019). Additionally, we thank our anonymous referee from $A p J$ for carefully reading and critiquing our paper. This work was supported in part by a grant from the Navajbai Ratan Tata trust.

\section{ORCID iDs}

Javed Rana @ https://orcid.org/0000-0001-5605-1809

\section{References}

Aasi, J., Abbott, B. P., Abbott, R., et al. 2015, CQGra, 32, 074001 Abbott, B. P., Abbott, R., Abbott, T. D., et al. 2017a, PhRvL, 119, 161101

Abbott, B. P., Abbott, R., Abbott, T. D., et al. 2017b, Natur, 551, 85

Abbott, B. P., Abbott, R., Abbott, T. D., et al. 2017c, ApJL, 850, L39 Abbott, B. P., Abbott, R., Abbott, T. D., et al. 2017d, ApJL, 850, L40 Abbott, B. P., Abbott, R., Abbott, T. D., et al. 2017e, ApJL, 851, L16 Abbott, B. P., Abbott, R., Abbott, T. D., et al. 2018, LRR, 21, 3 Acernese, F., Agathos, M., Agatsuma, K., et al. 2014, CQGra, 32, 024001 Andreoni, I., Ackley, K., Cooke, J., et al. 2017, PASA, 34, e069

Arcavi, I., Hosseinzadeh, G., Howell, D. A., et al. 2017, Natur, 551, 64 Ascenzi, S., Coughlin, M., Dietrich, T., et al. 2019, MNRAS, 486, 672

Astropy Collaboration, Robitaille, T. P., Tollerud, E. J., et al. 2013, A\&A, 558, A 33

Barnes, J., \& Kasen, D. 2013, ApJ, 775, 18

Bellm, E. C. 2014, The Third Hot-wiring the Transient Universe Workshop, ed. P. R. Wozniack et al., 27, http://www.slac.stanford.edu/econf/ C131113.1/papers/bellm.pdf

Chambers, K. C., Magnier, E. A., Metcalfe, N., et al. 2016, arXiv: 1612.05560

Chan, M. L., Hu, Y.-M., Messenger, C., Hendry, M., \& Heng, I. S. 2017, ApJ, 834,84

Chornock, R., Berger, E., Kasen, D., et al. 2017, ApJL, 848, L19

Cook, D. O., Kaliwal, M. M., Van Sistene, A., et al. 2017, arXiv:1710.05016

Cornish, N. J., \& Littenberg, T. B. 2015, CQGra, 32, 135012

Coughlin, M., \& Stubbs, C. 2016, ExA, 42, 165

Coughlin, M. W., Tao, D., Chan, M. L., et al. 2018, MNRAS, 478, 692

Coulter, D. A., Foley, R. J., Kilpatrick, C. D., et al. 2017, Sci, 358, 1556

Cowperthwaite, P. S., Berger, E., Villar, V. A., et al. 2017, ApJL, 848, L17

Dálya, G., Galgóczi, G., Dobos, L., et al. 2018, MNRAS, 479, 2374

Drout, M. R., Piro, A. L., Shappee, B. J., et al. 2017, Sci, 358, 1570

Essick, R., Vitale, S., Katsavounidis, E., Vedovato, G., \& Klimenko, S. 2015, ApJ, 800, 81

Evans, P. A., Cenko, S. B., Kennea, J. A., et al. 2017, Sci, 358, 1565

Gehrels, N., Cannizzo, J. K., Kanner, J., et al. 2016, ApJ, 820, 136

Ghosh, S., Bloemen, S., Nelemans, G., Groot, P. J., \& Price, L. R. 2016, A\&A, 592, A82

Ghosh, S., \& Bose, S. 2013, arXiv:1308.6081

GROWTH 2019a, Global Relay of Observatories Watching Transients Happen, http://growth.caltech.edu/

GROWTH 2019b, GROWTH-India, https://sites.google.com/view/growth india/first-light

Gutin, G., Yeo, A., \& Zverovich, A. 2002, Discrete Applied Mathematics, 117,81

Hardie, R. H. 1962, Astronomical Techniques (Chicago, IL: Univ. Chicago Press)

Hu, L., Wu, X., Andreoni, I., et al. 2017, Science Bulletin, 62, 1433

Ivezić, Z., Kahn, T., Tyson, J. A., et al. 2019, ApJ, 873, 111

Kasen, D., Fernandez, R., \& Metzger, B. 2015, MNRAS, 450, 1777

Kasliwal, M. M., Nakar, E., Singer, L. P., et al. 2017, Sci, 358, 1559

Kilpatrick, C. D., Foley, R. J., Kasen, D., et al. 2017, Sci, 358, 1583

Klimenko, S., Vedovato, G., Drago, M., et al. 2016, PhRvD, 93, 042004

Lipunov, V., Gorbovskoy, E., Kornilov, V. G., et al. 2017, ApJL, 850, L1

Max-Moerbeck, W. 2014, Proc. SPIE, 9149, 914925

McCully, C., Hiramatsu, D., Howell, D. A., et al. 2017, ApJL, 848, L32

Metzger, B. D. 2017, LRR, 20, 3

Metzger, B. D., \& Berger, E. 2012, ApJ, 746, 48

Munkres, J. 1957, SIAM, 5, 32

Nissanke, S., Kasliwal, M., \& Georgieva, A. 2013, ApJ, 767, 124

Petrillo, C. E., Dietz, A., \& Cavaglia, M. 2013, ApJ, 767, 140

Pian, E., D'Avanzo, P., Benetti, S., et al. 2017, Natur, 551, 67

Rana, J., Singhal, A., Gadre, B., Bhalerao, V., \& Bose, S. 2017, ApJ, 838, 108

Salafia, O. S., Colpi, M., Branchesi, M., et al. 2017, ApJ, 846, 62

Shanks, T., Metcalfe, N., Chehade, B., et al. 2015, MNRAS, 451, 4238

Shappee, B. J., Simon, J. D., Drout, M. R., et al. 2017, Sci, 358, 1574

Singer, L. P., Price, L. R., Farr, B., et al. 2014, ApJ, 795, 105

Smartt, S. J., Chen, T.-W., Jerkstrand, A., et al. 2017, Natur, 551, 75

Tanvir, N. R., Levan, A. J., Gonzalez-Fernandez, C., et al. 2017, ApJL, $848, \mathrm{~L} 27$ 